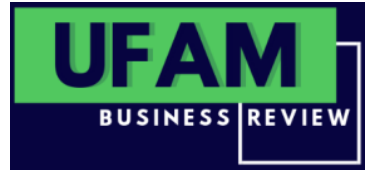

UFAM BUSINESS REVIEW

http://www.periodicos.ufam.edu.br/ufambr

Manaus, AM, Brasil, v. 3, n. 1, art. 2, pp. 21-38, janeiro/junho, 2021.

\title{
Investimento em Pesquisa e Desenvolvimento de Empresas de Alta e Média-Alta Tecnologia do Brasil
}

Investment in Research and Development of Brazil High and Medium Technology Companies

Inversión en Investigación y Desarrollo de las Empresas de Alta y Media-Alta Tecnología de Brasil

Mateus Muller ${ }^{1}$

mateusmuller159@hotmail.com https://orcid.org/0000-0002-2861-7091 http://lattes.cnpq.br/8053800094452987

Cristiane Canton ${ }^{1}$ cantoncristiane@gmail.com https://orcid.org/0000-0002-4074-435X http://lattes.cnpq.br/0677246806477561

Adriana Kroenke Hein ${ }^{1}$ akroenke@furb.br https://orcid.org/0000-0001-6625-3017 http://lattes.cnpq.br/0290174123868722

Fundação Universidade Regional de Blumenau, FURB, Brasil ${ }^{1}$

Recebido em: 31/10/2019 / Revisão: 01/11/2019 / Aprovado em: 18/02/2021

Editores responsáveis: Prof. Dr. Antônio Giovanni Figliuolo Uchôa e Prof. Dr. Jonas Fernando Petry Processo de Avaliação: Double Blind Review DOI: https://10.47357/ufambr.v3i1.6658 


\title{
Resumo
}

O avanço tecnológico incentiva cada vez mais o investimento em Pesquisa e Desenvolvimento $(P \& D)$, prática essa que influência a empresa de diversas maneiras, seja na liquidez, no endividamento e/ou na rentabilidade, tornando-se assim, um ponto de suma importância na gestão. A pesquisa, por abordar investimentos em $\mathrm{P} \& \mathrm{D}$, focou-se em empresas que, devido a sua área de atuação, são mais propensas a realizar tais investimento, sendo o caso das empresas de alta e média-alta tecnologia, classificadas segundo o IBGE. Ainda, evidencia-se a utilização do método Technique for Order of Preference by Similarity to Ideal Solution (TOPSIS) para classificação das companhias com base nos seus indicadores econômico-financeiros, de forma a verificar a diferença no investimento em $\mathrm{P} \& \mathrm{D}$ por parte das empresas com melhores indicadores. Os dados analisados referem-se ao período de 2013 a 2017 de treze companhias. O cálculo do TOPSIS, por meio de pesos, demonstrou que as variáveis de liquidez e rentabilidade possuem maior importância na análise para a colocação no ranking, evidenciando a importância dessas variáveis. Posteriormente, ao analisar o resultado do ranking com o investimento em $\mathrm{P} \& \mathrm{D}$, a correlação de Spearman indica que posições mais elevadas, ou seja, empresas com melhores indicadores econômico-financeiros, investem mais em P\&D. Esse resultado perpassa por similaridades encontradas na literatura que destacam a P\&D associada a vantagem competitiva, crescimento econômico a longo prazo e manutenção de alto padrão de lucros. $\mathrm{O}$ estudo contribui com a literatura analisando o contexto brasileiro de investimento em P\&D, destacando a sua relação com indicadores econômico-financeiros.

Palavras-chave: Pesquisa e Desenvolvimento. Empresas de Alta e Média-alta tecnologia. Indicadores econômico-financeiros, TOPSIS.

\section{Investment in Research and Development of Brazil High and Medium Technology Companies}

\begin{abstract}
Technological advancement increasingly encourages investment in Research and Development (R\&D), a practice that influences the company in various ways, whether in liquidity, indebtedness and / or profitability, thus becoming a very important point in management. The research, by addressing investments in $\mathrm{R} \& \mathrm{D}$, focused on companies that, due to their area of expertise, are more likely to make such investments, such as high and medium-high technology companies, classified according to the IBGE. Also, the use of the Technique for Order of Preference by Similarity to Ideal Solution (TOPSIS) method is used to classify companies based on their economic and financial indicators, in order to verify the difference in R\&D investment by companies with best indicators. The data analyzed refer to the period from 2013 to 2017 of thirteen companies. The TOPSIS calculation, using weights, demonstrated that the liquidity and profitability variables are more important in the analysis for ranking, highlighting the importance of these variables. Subsequently, when analyzing the ranking result with R\&D investment, Spearman's correlation indicates that higher positions, ie companies with better economic and financial indicators, invest more in $\mathrm{R} \& \mathrm{D}$. This result permeates similarities found in the literature that highlight $\mathrm{R} \& \mathrm{D}$ associated with competitive advantage, long-term economic growth and maintenance of high profit standards. The study contributes to the literature analyzing the Brazilian context of investment in $R \& D$, highlighting its relationship with economic and financial indicators.
\end{abstract}


Keywords: Research and Development. High and medium-high technology companies. Economic and financial indicators, TOPSIS.

\section{Inversión en Investigación y Desarrollo de las Empresas de Alta y Media-Alta Tecnología de Brasil}

\section{Resumén}

El avance tecnológico alienta cada vez más la inversión en Investigación y Desarrollo (I + D), una práctica que influye en la empresa de varias maneras, ya sea en liquidez, endeudamiento y / o rentabilidad, convirtiéndose así en un punto muy importante en gestión La investigación, al abordar las inversiones en I + D, se centró en empresas que, debido a su área de especialización, tienen más probabilidades de realizar tales inversiones, como empresas de tecnología alta y media-alta, clasificadas según el IBGE. Además, el uso de la técnica para el método de orden de preferencia por similitud con la solución ideal (TOPSIS) se utiliza para clasificar las empresas en función de sus indicadores económicos y financieros, con el fin de verificar la diferencia en la inversión en I + D de las empresas con mejores indicadores Los datos analizados se refieren al período de 2013 a 2017 de trece empresas. El cálculo TOPSIS, utilizando ponderaciones, demostró que las variables de liquidez y rentabilidad son más importantes en el análisis para la clasificación, destacando la importancia de estas variables. Posteriormente, al analizar el resultado del ranking con inversión en I + D, la correlación de Spearman indica que las posiciones más altas, es decir, las empresas con mejores indicadores económicos y financieros, invierten más en I + D. Este resultado impregna las similitudes encontradas en la literatura que destacan la I + D asociada con la ventaja competitiva, el crecimiento económico a largo plazo y el mantenimiento de altos estándares de ganancias. El estudio contribuye a la literatura que analiza el contexto brasileño de inversión en I + D, destacando su relación con los indicadores económicos y financieros.

Palabras clave: Investigación y desarrollo. Empresas de alta y media-alta tecnología. Indicadores económicos y financieros, TOPSIS. 


\section{INTRODUÇÃO}

A globalização da economia mundial trouxe vários desafios para as empresas do século XXI, com isso, para ser uma das companhias líderes de mercado há a necessidade de estar sempre à frente dos concorrentes. Consequentemente, percebeu-se que nos últimos 50 anos, tanto na realidade acadêmica quanto empírica, passou-se a considerar os investimentos em Pesquisa e Desenvolvimento (P\&D) como parâmetro (proxy) para inovação e progresso tecnológico das empresas (Koh \& Reeb, 2015).

Dessa maneira, observa-se a importância da $\mathrm{P} \& \mathrm{D}$, sendo fundamental para as empresas, levando a implementação de produtos e processos tecnológicos novos ou melhorados (Gonçalves e Lemes, 2018), auxiliando a companhia a possuir um diferencial competitivo e aumentando seu poder de mercado (Aghion, Bechtold, Cassar, \& Herz, 2014). Dessa maneira, percebeu-se os benefícios que são advindos do investimento nessa prática.

No contexto norte-americano, japonês e europeu, há uma onda de crescimento em investimentos em P\&D, principalmente em áreas de maior tecnologia e indústrias baseadas em ciências como a biotecnologia, telecomunicações e softwares, além, é claro, da concorrência a nível internacional, o que incentiva o aumento da importância dos gastos em P\&D (Akcali \& Sismanoglu, 2015). Por se tratarem das principais economias mundiais, as demais tendem a seguir a mesma perspectiva, indo ao encontro dos resultados desses países. No entanto, as inovações geralmente estão relacionadas a criação de emprego em economias baseadas no conhecimento (Coad, 2018), o que pode não ser a realidade do país, principalmente no contexto de países subdesenvolvidos.

No Brasil, o Instituto Brasileiro de Geografia e Estatística (IBGE, 2003), por meio da Pesquisa Industrial Anual (PIA), realizou um estudo em que caracterizou e classificou os setores da economia brasileira de acordo com a sua tecnologia e indicadores econômico-financeiros, de forma a verificar os setores mais inovadores. Por meio dessa classificação, nota-se que, assim como no contexto mundial, as empresas caracterizadas como mais inovadoras, que nesse caso significa que mais investem em P\&D, são as relacionadas a itens de tecnologia e informática, telecomunicações e refino de petróleo. No entanto, esses setores são minoria na bolsa de valores Brasil Bolsa Balcão (B3).

No país, apesar de se encontrar várias empresas relacionadas a inovação, tem-se a predominância de empresas ligadas a classificação de média-baixa e baixa tecnologia. No entanto, essas não são as companhias mais propensas a realizar investimentos em $P \& D$, já que as empresas de alta e média alta tecnologia normalmente investem mais devido ao setor e necessidade quanto a produtos e serviços (Landry \& Callimaci, 2003). Dessa forma, a questão norteadora do estudo é: qual a relação da classificação das empresas pelos seus indicadores econômico-financeiros e o investimento em $P \& D$ ? Portanto, o objetivo é verificar se as melhores empresas do ranking formulado com base nos indicadores econômicos financeiros apresentam maior relação com investimento em P\&D.

O estudo justifica-se pela importância do tema $\mathrm{P} \& \mathrm{D}$, pois esse tipo de investimento é "o motor do crescimento econômico", determinando o crescimento econômico de longo prazo das empresas (Akcigit \& Kerr, 2015). Assim, a inovação gerada por esse investimento é tida como um componente crítico na avalição de longo prazo (Koh \& Reeb, 2015) e como vantagem competitiva (Aghion et al., 2014). Portanto, pode-se afirmar que compreender a importância da 
P\&D é fundamental para Stakeholders e Shareholders, pois influencia diretamente no crescimento da companhia em vendas e valor de mercado.

No contexto brasileiro, devido a ser um país em desenvolvimento, observa-se que os valores investidos em inovação são baixos, em que se estima uma relação de cerca de $1 \%$ do Produto Interno Bruto (PIB) sendo investido em P\&D (MCTI, 2016), um valor menor se comparado a países desenvolvidos. Também se identifica que o tipo de indústria influencia a inovação (Coad, 2018), por isso, visualizou-se que, no Brasil, dois grupos de empresas diferenciam-se, sendo estas as companhias de alta e média-alta tecnologia, em virtude dos seus setores de atuação (Landry \& Callimaci, 2003), logo, a proporção de investimento em P\&D dessas empresas é maior, por isso a análise busca verificar esse contexto.

Os resultados evidenciam que dentre os indicadores que constam no estudo, os de Liquidez (Corrente e Geral) são os principais no cálculo da classificação no ranking pelo método Technique for Order of Preference by Similarity to Ideal Solution (TOPSIS), tanto no cálculo com peso pelo método CRITIC quanto sem. Esses indicadores são ressaltados devido a possuírem relevância na capacidade de investimento em $\mathrm{P} \& \mathrm{D}$, pois permitem a empresa não buscar capital de terceiros, que é oneroso. Assim, as companhias na primeira metade do ranking, ou seja, com os melhores indicadores, investem um percentual maior em P\&D proporcional ao seu Ativo Total, se comparada com empresas de classificação mais baixa, isso mostra que mesmo em um contexto de setores mais propensos ao investimento em $\mathrm{P} \& \mathrm{D}$, indicadores melhores estão associados ao maior investimento.

O estudo contribui para a teoria, relacionando o ranking baseado em indicadores econômicofinanceiros elaborado pelo método TOPSIS com o gasto em P\&D, devido esse tipo de investimento necessitar de valores substanciais e sequenciais para gerar benefícios econômicos futuros, agregando a relação à temática de P\&D. É empírica, evidenciando para as companhias os efeitos relacionados a prática do investimento em inovação. E é social, em virtude de que investimentos em $\mathrm{P} \& \mathrm{D}$ gerarem benefícios econômicos para as empresas e países, aumentando o PIB e gerando novos empregos e soluções tecnológicas que são utilizadas pela sociedade.

O estudo divide-se nas seguintes seções: Introdução; Referencial Teórico; onde visa embasar os indicadores utilizados no ranking e sua relação com o investimento em P\&D; Enquadramento Metodológico, a fim de compreender de que maneira se procedeu o estudo; Análise e Discussão dos Resultados, onde são descritos os achados da pesquisa; Considerações Finais e Referências.

\section{REFERENCIAL TEÓRICO}

Nesta seção, trata-se de P\&D e fatores inerentes a esse investimento, de forma a compreender seus possíveis impactos nas empresas. Na sequência, esses fatores são dimensionados por meio de indicadores econômico-financeiros para testar a relação esperada.

\subsection{Pesquisa e Desenvolvimento (P\&D)}

O investimento em P\&D tem como objetivo a inovação que pode introduzir novos processos ou produtos. Os efeitos relacionados a produtividade, advinda da inovação, tem sido um assunto de grande interesse por parte de pesquisadores e formuladores de políticas (Ugur, Trushin, \& Solomon, 2016). A ampliação da produtividade está ligado principalmente ao aumento de resultados da companhia, que por meio da introdução de inovações a longo prazo, mantêm um 
alto padrão de lucro (Love, Roper, \& Du, 2009). A relação de lucro ou desempenho com P\&D é visualizada em diversos estudos (Doh \& Kim, 2014; Guo, Guo, \& Jiang, 2017; Huang \& Hou, 2019; Jung, Hwang, \& Kim, 2018; Radas, Anić, Tafro, \& Wagner, 2015; Ugur et al., 2016).

No entanto, para que a inovação, por meio da $\mathrm{P} \& \mathrm{D}$, atinja seus objetivos, dois pontos devem ser observados. Primeiro, os insumos inerentes ao processo não garantem a inovação em si, mesmo que as empresas direcionem uma proporção significativa de seus recursos, em virtude de que a inovação trata-se de um processo cheio de riscos e incertezas, dessa forma, não é possível antecipar os seus resultados (Cowling, Liu, Ledger, \& Zhang, 2015). Segundo, a maior parte do investimento em $\mathrm{P} \& \mathrm{D}$ consiste em pagamentos salariais a pesquisadores, engenheiros e demais trabalhadores da área, que demandam uma grande quantidade de treinamento específico para realização de suas atividades (Brown \& Petersen, 2011), ou seja, investimentos que podem ser perdidos por saída ou troca de funcionários. Observam-se nuances da P\&D que influenciam a decisão dos administradores no momento de investir nessa prática.

O financiamento acaba por se tornar outro ponto de fundamental importância para empresas que investem em P\&D. Destaca-se que o financiamento insuficiente dessa atividade é, quase sempre, uma ameaça potencial ao sucesso e sobrevivência de um empreendimento que investe em P\&D (Cowling et al., 2015). Um investimento mal realizado, acaba por afetar os resultados da organização no momento e no seu futuro, pois além do gasto momentâneo, não haverá retorno de um valor que poderia ter sido aplicado em outra atividade da empresa. Por meio da literatura, alguns exemplos de formas de financiamentos para P\&D são enfatizadas, sendo elas o financiamento interno, que ocorre por meio do fluxo de caixa, e financiamento externo, seja por emissão de dívidas ou de ações (Guney, Karpuz, \& Ozkan, 2017).

Ambas as formas de financiamento possuem repercussão na organização. Visualiza-se que, referente ao financiamento interno por meio do fluxo de caixa, o investimento em P\&D possui implicação direta com liquidez corporativa (Brown \& Petersen, 2011). Teoricamente, as empresas necessitam de reservas (liquidez de caixa) ou linhas de crédito para amenizar efeitos de choques de liquidez (Holmström \& Tirole, 1998). Essa forma de financiamento é útil por não exigir garantias, os retornos positivos para os investidores não são limitados e não aumenta a probabilidade de dificuldade financeira (Guney et al., 2017). Portanto, a forma mais eficiente de financiamento da $\mathrm{P} \& \mathrm{D}$, conforme a literatura, seria com base na capacidade de pagamento próprio da empresa, por meio da sua liquidez, sem necessitar de capital externo que é oneroso.

Empiricamente, no contexto europeu, o fluxo de caixa possui um efeito positivo e significativo no investimento em P\&D (Brown, Martinsson, \& Petersen, 2012) e o mesmo resultado pode ser verificado no contexto norte-americano (Brown \& Petersen, 2015), atestando que em países desenvolvidos, as empresas acabam preferindo financiar o P\&D internamente. No entanto, é preciso observar que manter grandes reservas de caixa, no caso do financiamento interno, é dispendioso para a organização, e, nos casos de esgotamento do caixa, não se é possível continuar o investimento (Brown \& Petersen, 2011), o que levaria a uma paralisação do investimento e a possível perda de funcionários e dos resultados obtidos até o momento.

Além do financiamento interno, comentado anteriormente, a empresa também pode optar pelo financiamento externo. No entanto, há uma diferença entre ambos, a qual se trata do financiamento externo ser mais caro que o interno, isso devido aos riscos inerentes a incerteza do projeto, a assimetria de informação no investimento em $\mathrm{P} \& \mathrm{D}$, nos potenciais produtos e retornos financeiros esperados, que faz com que essa prática seja sensível a choques externos e flutue (Guney et al., 2017; Kang, Baek, \& Lee, 2017). Outra razão é que o financiamento por 
meio de dívida pode levar a organização a enfrentar dificuldades financeiras, principalmente em empresas intensivas em P\&D (Cornell \& Shapiro, 1988; Opler \& Titman, 1994). Dessa forma, o financiamento externo seria uma prática utilizada por companhias que não dispõem de liquidez.

Referente ao resultado do investimento em $\mathrm{P} \& \mathrm{D}$, tem-se, conforme a literatura, que há um aumento no desempenho da organização e/ou no lucro. Eberhart, Maxwell, \& Siddique, (2004) descobriram que as empresas que aumentam seus gastos com $\mathrm{P} \& \mathrm{D}$ reportam desempenho operacional futuro significativamente positivo. Karjalainen, (2008), acredita que os investimentos em P\&D têm impactos positivos no nível de rentabilidade futura da empresa. Portanto, espera-se que companhias que investem sucessivamente em P\&D há anos possuam um resultado melhor, devido a essa prática e, com o maior lucro, possuam mais fluxo de caixa para continuar investindo nessa prática, diminuindo dependência do financiamento externo.

De forma geral, percebe-se que os efeitos do investimento em $\mathrm{P} \& \mathrm{D}$ em curto prazo são relacionados a diminuição dos recursos que seriam utilizados de outras formas dentro da organização. Porém, os resultados futuros esperados são de aumento de rentabilidade e, consequentemente, no fluxo de caixa (liquidez). Assim, espera-se que empresas que possuam os melhores indicadores, ou seja, melhor classificadas no ranking, invistam mais em P\&D, devido a colherem resultados de investimentos passado.

Para análise da variável de P\&D neste estudo, verifica-se os seus gastos em empresas da amostra que divulgaram a informação por meio de suas demonstrações financeiras. Em virtude de diferenças demográficas das empresas, decidiu-se escalonar o valor de investimento em P\&D pelo ativo total da companhia, devido a, teoricamente, empresas maiores terem mais condições de investir e assim, amenizando problemas de tamanho.

\subsection{Indicadores}

Os indicadores financeiros são formas objetivas de acompanhar o desempenho das companhias e permitir aos investidores e parceiros a avaliação do estado em que se encontram informações importantes sobre a empresa (Assaf Neto, 2014). São encontrados na literatura artigos que utilizam dos indicadores econômico-financeiros para avaliação das empresas e teste de relações. Os indicadores que fazem parte do estudo estão na tabela 01.

Tabela 01: Indicadores Econômico-Financeiros.

\begin{tabular}{c|c|c|c}
\hline \multirow{2}{*}{ Grupo } & Variável & Fórmula & Autores \\
& Liquidez Corrente (LC) & $\frac{A C}{P C}$ & $\begin{array}{c}\text { Viglioni, Carvalho, Benedicto, } \\
\text { \& Do Prado, 2018) }\end{array}$ \\
\cline { 2 - 4 } & Liquidez Geral (LG) & $\frac{A C+A R L P}{P C+P E L P}$ & (Viglioni et al., 2018) \\
\hline \multirow{2}{*}{ Endividamento } & Endividamento (END) & $\frac{\text { Capital de Terceiros }}{A T}$ & $\begin{array}{c}\text { (Tonolli, Rover, \& Ferreira, } \\
\text { 2017) }\end{array}$ \\
\hline \multirow{2}{*}{ Rentabilidade } & Return of Assets (ROA) & $\frac{L L}{A T}$ & $\begin{array}{c}\text { (Tonolli et al., 2017; Viglioni et } \\
\text { al., 2018) }\end{array}$ \\
\cline { 2 - 4 } & Return of Equity (ROE) & $\frac{L L}{P L}$ & $\begin{array}{c}\text { (Viglioni et al., 2018) } \\
\text { Giro do Ativo }\end{array}$ \\
\cline { 2 - 4 } & Giro do Ativo (GA) & $\frac{V L}{A T}$ & (Viglioni et al., 2018) \\
\hline
\end{tabular}


Legenda: AC - Ativo Circulante; ARLP - Ativo Realizável a Longo Prazo; AT - Ativo Total; LL - Lucro Líquido; PC - Passivo Circulante; PELP - Passivo Exigível a Longo Prazo; PL - Patrimônio Líquido; e VL - Vendas Líquidas.

Fonte: Dados da pesquisa.

O indicador de LC apresenta a capacidade de pagamento a curto prazo, no entanto, esse cálculo não demonstra a qualidade dos itens e a sincronização dos recebimentos e pagamentos (Marion, 2012). A LC também é tida como o melhor indicador da situação de liquidez da empresa (Iudícibus, 2012).

A LG atesta a capacidade de pagamento da companhia no longo prazo, considerando, conjuntamente, os valores a curto prazo, que se converterão em dinheiro, sendo relacionado com o total de dívidas assumidas (Marion, 2012). No entanto, pelo indicador ser muito amplo quanto aos prazos, acaba empobrecendo o sentido e a utilização do quociente (Iudícibus, 2012), devido que os valores do indicador podem ser positivos, porém, se o recebimento for em um prazo maior que o pagamento, haverá problema de liquidez.

O indicador de endividamento mostra o percentual do ativo que é financiado pelos recursos que advém de terceiros (Iudícibus, 2012). No contexto de países desenvolvidos, o indicador é mais elevado, podendo representar $60 \%$, isso em virtude da competitividade, assim, os recursos próprios não são suficientes para atender a renovação de Ativos e recorre-se a Capitais de terceiros (Marion, 2012).

Os indicadores de rentabilidade (ROA e ROE) evidenciam a medição da rentabilidade por funções de investimentos, sendo elas o Ativo e o Capital Próprios. No primeiro caso, representa que a administração adequada do Ativo proporciona um maior retorno para a empresa. No segundo, os investidores podem analisar o retorno em relação aos seus valores investidos na companhia (Marion, 2012). Portanto, as métricas de rentabilidade apresentam informações para dois públicos diferentes e importantes da empresa.

O GA indica o quanto o ativo é capaz de gerar em vendas reais, ou seja, uma forma de medir a eficiência da gestão no uso dos seus ativos (Marion, 2012). Dessa forma, quanto maior o giro em virtude das vendas, maiores são as chances de cobrir as despesas com uma boa margem de lucro (Iudícibus, 2012).

Todos os indicadores relacionados ao ranking possuem funções de análise da empresa e representam pontos vitais, como a capacidade de pagamento de obrigações (indicadores de liquidez), financiamento das empresas (endividamento) e análises quanto a eficiência na gestão das atividades (indicadores de rentabilidade e giro do ativo). A relação desses itens com P\&D está disposto no próximo tópico.

No contexto de empresas de tecnologia, semelhante ao escolhido neste estudo, Viglioni et al. (2018), utilizam os indicadores econômico-financeiros como determinantes para analisar fusões e aquisições no Brasil. Os resultados obtidos na pesquisa auferem que indicadores como LG, ROA e ROE, que são utilizados neste estudo, não são caracterizados como determinantes na relação analisada. Em contrapartida, capital de terceiros e tamanho da empresa são as principais variáveis que determinam a decisão de fusão ou aquisições.

Agregando a análise fatores ambientais, Tonolli, Rover e Ferreira (2017) investigaram a influência de investimentos ambientais e dos indicadores econômico-financeiros na seleção de empresas para compor o índice de sustentabilidade empresarial. Os resultados evidenciaram 
que indicadores como tamanho, ROA, grau de endividamento e o investimento ambiental influenciam a empresa a compor o índice.

\section{PROCEDIMENTOS METODOLÓGICOS}

As pesquisas no campo de investigação das ciências sociais aplicadas podem ser classificadas metodologicamente quanto aos seus objetivos, estratégia de coleta de dados e em relação à abordagem de análise (Beuren, 2004; Collis \& Hussey, 2005). Dessa forma, delineia-se essa pesquisa como descritiva em relação ao objetivo, pois pretende descrever as empresas por meio dos indicadores econômico-financeiros das companhias de alta e média-alta tecnologia da B3 e a sua relação com o investimento em $\mathrm{P} \& \mathrm{D}$, configura-se como análise documental, visto que os dados utilizados para cálculo das variáveis foram obtidos por meio da base de dados Economática ${ }^{\circledR}$, e de abordagem quantitativa na análise dos dados.

\subsection{População e Amostra}

No Brasil, o Instituto Brasileiro de Geografia e Estatística (IBGE, 2003), por meio da Pesquisa Industrial Anual (PIA), realizou um estudo em que caracterizou e classificou os setores da economia brasileira de acordo com a sua tecnologia e indicadores econômico-financeiros, de forma a verificar os setores mais inovadores. Por meio dessa classificação, nota-se que assim como no contexto mundial caracterizado por Akcali e Sismanoglu (2015), as empresas descritas como mais inovadoras, utilizado como proxy o investimento em $\mathrm{P} \& \mathrm{D}$, são as relacionadas a itens de tecnologia e informática, telecomunicações e refino de petróleo. No entanto, esses setores são minoria na Brasil Bolsa Balcão (B3), se comparado com outros setores. Os dados relativos aos indicadores foram coletados a partir Economática enquanto que os dados de P\&D foram coletados da base de dados Thomson Reuters.

A população referente aos setores relacionados ao uso de alta e média-alta tecnologia é composta, no total, por 59 companhias. Porém, desse total, a falta de dados reduziu $78 \%$ da população devido à falta da variável de $\mathrm{P} \& \mathrm{D}$, sendo essa de divulgação limitada no contexto brasileiro. Dessa forma, a amostra é de 13 empresas, ou seja, $22 \%$ da população. O mesmo percalço da quantidade amostral é encontrado por Gonçalves e Lemes (2018) ao analisar a mesma população, empresas de alta e média-alta tecnologia no Brasil. No entanto, os autores destacam a importância dos setores pertencentes a essa população. A divisão de setor referente a alta e média-alta tecnologia está contida na tabela 02 .

Tabela 02: Classificação empresas de Alta e Média-Alta Tecnologia

\begin{tabular}{l|l}
\hline Classificação & Subsetor Bovespa \\
\hline \multirow{4}{*}{$\begin{array}{l}\text { Alta Tecnologia } \\
\text { (9 empresas) }\end{array}$} & Automóveis e motocicletas \\
\cline { 2 - 2 } & Computadores e equipamentos \\
\cline { 2 - 2 } & Material de transporte \\
\cline { 2 - 2 } $\begin{array}{l}\text { Média-Alta Tecnologia } \\
(4 \text { empresas) }\end{array}$ & Petróleo, gás e biocombustíveis \\
\cline { 2 - 2 } & Madeira e papel \\
\cline { 2 - 2 } & Químicos equipamentos \\
\hline
\end{tabular}

Fonte: IBGE (2003).

Portanto, após as exclusões, os setores que possuem empresas na pesquisa são os elencados na tabela 02. Posteriormente, foram elaborados os rankings pelo desempenho econômicofinanceiro, pelo método TOPSIS e, em seguida, analisado se o melhor posicionamento no 
ranking se relaciona com maior investimento em $\mathrm{P} \& \mathrm{D}$, calculado utilizando-se do teste estatístico correlação de Spearman. Adicionalmente, optou-se por verificar o efeito defasado da P\&D nos indicadores futuros das empresas. Ambos os métodos são citados a seguir.

\subsection{Método TOPSIS}

A primeira vez que se utilizou o método TOPSIS foi em 1981, por Hwang e Yoon, tendo como ideia principal o conceito vindo do compromisso da solução alternativa escolhida para ter a distância mais próxima de uma solução ideal positiva (solução ótima) e ter maior distância da solução ideal negativa (solução não ótima) (Yoon \& Hwang, 1995). Esse método se baseia no conceito a qual refere-se a alternativa melhor escolhida não tem somente a distância mais curta da solução positiva ideal, mas também a maior distância da solução ideal, assim, esse conceito é amplamente utilizado para resolver problemas práticos de decisão.

Observa-se na literatura nacional alguns estudos que utilizaram o TOPSIS em sua análise, como Hein, Degenhart, Vogt, Kroenke e Campestrini (2015) que utilizam esse método para avaliação das empresas listadas na IBRX-100, Degenhart, Vogt, Hein, Da Rosa e Fank (2015) que formulam um ranking de evidenciação de impactos ambientes em empresas brasileiras. No âmbito internacional, o estudo de Balcerzak e Peitrzak (2016) tem por objetivo examinar o contexto de desenvolvimento sustentável europeu, utilizando-se do método TOPSIS para avaliar objetos em termos de fenômenos econômicos multidimensionais. O índice sintético dinâmico que descreve o nível relativo de desenvolvimento sustentável dos países foi criado, que permitiu propor uma classificação dos países e agrupá-los em subconjuntos homogêneos.

Passos para calcular o método TOPSIS:

(1) Matriz de decisão: as colunas representam os critérios (n) de decisão e as linhas as alternativas (m).

$x_{i j}=\left[\begin{array}{cccc}x_{11} & x_{12} & \ldots & x_{1 n} \\ x_{21} & x_{22} & \ldots & x_{2 n} \\ \cdot & \cdot & \ldots & \cdot \\ x_{m 1} & x_{m 2} & \ldots & x_{m n}\end{array}\right]$

(2) Normalização da matriz.

$$
r_{i j}=\frac{x_{i j}}{\sqrt{\sum_{i=1}^{m} x_{i j}^{2}}}
$$

(3) Cálculo da matriz de decisão normalizada ponderada.

$y_{i j}=w_{1} r_{i j}$

(4) Cálculo da solução ideal positiva e negativa: a solução positiva $A^{+}$ideal e a solução $A^{-}$negativa ideal podem ser determinadas com base na classificação ponderada normalizada (Yij), como segue:

$$
\begin{aligned}
& A^{+}=\left(y_{1}^{+}, y_{2}^{+}, \ldots y_{n}^{+}\right) \\
& A^{-}=\left(y_{1}^{-}, y_{2}^{-}, \ldots y_{n}^{-}\right)
\end{aligned}
$$


(5) Cálculo da distância com a solução ideal: distância é uma alternativa Ai com uma solução ideal positiva é assumida da seguinte forma

$D_{i}^{+}=\sqrt{\sum_{j=1}^{n}\left(y_{i j}-y_{j}^{+}\right)}$

Distância é a alternativa Ai com a solução ideal é assumida da seguinte forma:

$D_{i}^{-}=\sqrt{\sum_{j=1}^{n}\left(y_{i j}-y_{j}^{-}\right)}$

(6) Cálculo do valor de preferência: O valor de preferência para cada alternativa (Vi) é dado como:

$V_{i}=\frac{D_{1}^{-}}{D_{1}^{-}-D_{1}^{+}}$

Como o método TOPSIS ainda permite a utilização de pesos, utilizou-se o Criteria Importance Through Intercriteria Correlation (CRITIC), que emprega o desvio-padrão e correlação para determinação de pesos em problemas de Multiple Criteria Decision Making (MCDM), assim os pesos derivados incorporam intensidade de contraste e conflito que estão contidos na estrutura do problema de decisão. O método desenvolvido baseia-se na investigação analítica da matriz de avaliação para extrair toda a informação contida nos critérios de avaliação (Diakoulaki, Mavrotas, \& Papayannakis, 1995).

Posteriormente, com o objetivo de analisar o investimento em P\&D pelas companhias, com base nas empresas ranqueadas pelos seus indicadores econômico-financeiros, utilizou-se o teste estatístico correlação de Spearman. Esse teste estatístico avalia quão bem uma função monotônica arbitrária pode descrever a relação entre duas variáveis, sem realizar suposições quanto a distribuição de frequência das variáveis (Hauke \& Kossowski, 2011). Esse teste é utilizado devido ao seu coeficiente ser mais robusto para outliers do que o coeficiente da correlação de Pearson (Mukaka, 2012). Além disso, a correlação de Spearman é comumente utilizada em análises advindas de classificações, como neste caso, da utilização do método TOPSIS.

Para concluir, ainda se optou por utilizar uma regressão linear para verificar a relação entre o investimento em P\&D e o resultado futuro da companhia, verificado pelo índice resultante do TOPSIS. Essa análise embasa-se em efeitos discutido na literatura por, Eberhart et al. (2004), Huang e Hou (2019) e Karjalainen (2008) os quais discutem que a P\&D proporcionam benefícios a empresa em períodos subsequentes ao do investimento ser realizado.

\section{ANÁLISE DOS RESULTADOS}

O ranking foi determinado com base nos indicadores que constam no referencial teórico, com o total de 13 empresas classificadas como de alta e média-alta tecnologia. A classificação alcançada por cada empresa, seja pelo método sem pesos ou com peso, pelo CRITIC, é evidenciado na tabela 03 .

Tabela 03: Classificação método TOPSIS

\begin{tabular}{lllllllllll}
\hline EMPRESA & 2013 & $2013 \mathrm{C}$ & 2014 & $2014 \mathrm{C}$ & 2015 & $2015 \mathrm{C}$ & 2016 & $2016 \mathrm{C}$ & 2017 & $2017 \mathrm{C}$ \\
\hline
\end{tabular}


Investimento em Pesquisa e Desenvolvimento de Empresas de Alta e Média-Alta Tecnologia do Brasil

\begin{tabular}{|c|c|c|c|c|c|c|c|c|c|c|}
\hline QGEP Part (*) & 1 & 1 & 1 & 1 & 2 & 2 & 2 & 2 & 1 & 1 \\
\hline Ultrapar Part (*) & 2 & 3 & 4 & 4 & 3 & 3 & 3 & 4 & 4 & 4 \\
\hline Petro Rio SA $(*)$ & 3 & 2 & 2 & 2 & 1 & 1 & 1 & 1 & 2 & 2 \\
\hline Metal Leve SA (*) & 4 & 4 & 6 & 10 & 7 & 8 & 6 & 6 & 5 & 5 \\
\hline Fras Le SA $(*)$ & 5 & 5 & 5 & 5 & 8 & 7 & 4 & 3 & 7 & 8 \\
\hline Randon Part (*) & 6 & 6 & 3 & 3 & 4 & 4 & 5 & 5 & 3 & 3 \\
\hline Suzano Papel & 7 & 7 & 9 & 7 & 9 & 9 & 8 & 8 & 10 & 9 \\
\hline Suzano Hold & 8 & 8 & 10 & 9 & 11 & 10 & 10 & 10 & 11 & 12 \\
\hline Embraer SA $(*)$ & 9 & 9 & 7 & 6 & 10 & 6 & 9 & 9 & 8 & 6 \\
\hline Indústrias Romi SA & 10 & 10 & 8 & 8 & 5 & 5 & 7 & 7 & 6 & 7 \\
\hline Petrobras $(*)$ & 11 & 11 & 13 & 12 & 12 & 12 & 11 & 11 & 12 & 11 \\
\hline Braskem SA & 12 & 12 & 12 & 13 & 6 & 11 & 13 & 13 & 9 & 13 \\
\hline Itautec $(*)$ & 13 & 13 & 11 & 11 & 13 & 13 & 12 & 12 & 13 & 10 \\
\hline
\end{tabular}

Legenda: Anos acompanhados com a letra "C" referem-se ao cálculo com peso do CRITIC.

$(*)$ - Empresas de Alta Tecnologia

Fonte: Dados da pesquisa.

O primeiro ponto a se observar é que em nenhum ano, tanto na análise com e sem peso, uma empresa de média-alta tecnologia é caracterizada como a melhor, com base nos seus indicadores. A melhor colocação alcançada por uma companhia de média-alta tecnologia é a de quinto lugar pelas Indústrias Romi SA em 2015. As demais obtiveram colocações sempre abaixo da sexta colocação, mas em raros momentos de melhora dos seus indicadores.

Dentre as empresas que mais se destacaram nos rankings, a QGEP Part e a Petro Rio apresentam os maiores valores referentes aos indicadores de liquidez, seja LC ou LG. Esses indicadores são de extrema importância para a organização, já que por meio dela apresenta-se a capacidade de pagamento das obrigações a curto e longo prazo (Iudícibus, 2012; Marion, 2012). Além da importância geral para a empresa, a liquidez é diretamente vinculada ao financiamento do investimento em $\mathrm{P} \& \mathrm{D}$, sendo a forma mais eficiente, por não aumentar a probabilidade de dificuldade financeira, o que ocorre ao buscar capital de terceiros (Guney et al., 2017). Em contrapartida, companhias como a Itautec e a Petrobras, e especialmente a Braskem, com classificações mais baixas, apresentam os indicadores de liquidez inferiores ao encontrado para a média da amostra.

A partir do END, observa-se que as empresas da pesquisa possuem esse indicador com média que varia de 0,68 a 0,90 durante os cinco anos do estudo. Visualiza-se assim, que a maior parte do financiamento das companhias da pesquisa advém de terceiros (Iudícibus, 2012; Marion, 2012). Referente a Petro Rio e QGEP Part, as duas empresas que estiverem entre as melhores do ranking apresentaram um END abaixo da média, especialmente a Petro Rio, que em 2013, menos de $20 \%$ do seu ativo possuía origem de capital de terceiros. Esse indicador, quando alto, não necessariamente é ruim, variando conforme a estratégia da organização, sendo uma forma de aumentar as atividades da empresa. Porém, também representa um custo mais elevado do que o financiamento interno, mas nos casos de falta de liquidez, é uma forma da companhia manter investimentos, como por exemplo em $\mathrm{P} \& \mathrm{D}$, e não perder os resultados obtidos até o momento. Ainda, pode-se verificar que as empresas que apresentam os melhores indicadores de liquidez possuem, em contrapartida, o menor índice de endividamento e, segundo a literatura, a empresa opta por se utilizar de uma ou outra forma para o financiamento de P\&D, seja capital próprio ou de terceiros. 
Referente a rentabilidade, medida por ROA e ROE, os resultados mostram que várias empresas obtiveram resultados negativos. Devido aos resultados, é importante salientar o momento econômico brasileiro vivido durante os anos do estudo, principalmente 2015 e 2016, em que houve crise econômica. Observa-se, partir do ranking, que companhias como a Qpeg Part, que figuraram entre as principais colocações, possuem resultados positivos em ambos os indicadores, o que indica que elas estão proporcionando retorno para os administradores (por meio do ROA) e dos investidores (por meio do ROE). Quanto as empresas com indicadores menos representativos, como os casos da Braskem, Itautec e Petrobras, os indicadores de rentabilidade, na maioria dos períodos, aproximavam-se ou estavam abaixo de zero. Em virtude de tal, espera-se que essas empresas possuam menor capacidade de investimento por meio do capital próprio e necessite de capital de terceiros, o qual é mais oneroso.

O GA, que representa a eficiência do ativo para gerar vendas (Marion, 2012). Apesar das empresas Qpeg Part e Petro Rio terem ótimos resultados no ranking, o GA de ambas as companhias foram baixos e, se verificar o peso determinado pelo CRITIC, esse indicador é um dos menos representativos.

De forma a exemplificar melhor a importância da liquidez no ranking, observa-se que, quando há a estimação de pesos pelo método CRITIC, em que os pesos são calculados com base na correlação dos indicadores, a LC é o fator mais importante, ou seja, mais representativo dentro do ranking. Observa-se ainda, que a análise com e sem pesos possui algumas diferenças, principalmente nas companhias que estão em posições intermediárias, mas com menor intensidade nas companhias dos dois extremos (primeiras e últimas posições), o que auxilia na confirmação dos resultados do ranking.

Com o ranking construído, parte-se agora para a análise referente ao investimento em $\mathrm{P} \& \mathrm{D}$. Os valores percentuais do investimento escalonado pelo ativo total estão dispostos na tabela 04 .

Tabela 04: Investimento em P\&D escalonado pelo ativo total.

\begin{tabular}{lccccc}
\hline EMPRESA & $\mathbf{2 0 1 7}$ & $\mathbf{2 0 1 6}$ & $\mathbf{2 0 1 5}$ & $\mathbf{2 0 1 4}$ & $\mathbf{2 0 1 3}$ \\
\hline Braskem SA & $0,31 \%$ & $0,31 \%$ & $0,28 \%$ & $0,26 \%$ & $0,24 \%$ \\
\hline Embraer SA $\left.*^{*}\right)$ & $0,40 \%$ & $0,43 \%$ & $0,31 \%$ & $0,41 \%$ & $0,67 \%$ \\
\hline Fras Le SA $(*)$ & - & - & $0,58 \%$ & $0,68 \%$ & $0,64 \%$ \\
\hline Indústrias Romi SA & $1,45 \%$ & $1,77 \%$ & $1,39 \%$ & $1,54 \%$ & $1,34 \%$ \\
\hline Itautec $(*)$ & - & - & $0,02 \%$ & $0,13 \%$ & $0,02 \%$ \\
\hline Metal Leve SA $(*)$ & $3,46 \%$ & $3,33 \%$ & $3,46 \%$ & $2,70 \%$ & $2,75 \%$ \\
\hline Petro Rio SA $(*)$ & $0,06 \%$ & $0,07 \%$ & $0,12 \%$ & $0,47 \%$ & $2,44 \%$ \\
\hline Petrobras $(*)$ & $0,53 \%$ & $0,98 \%$ & $0,94 \%$ & $1,23 \%$ & $1,18 \%$ \\
\hline QGEP Part $(*)$ & $-0,07 \%$ & $1,91 \%$ & $1,28 \%$ & $1,38 \%$ & $2,68 \%$ \\
\hline Randon Part $(*)$ & - & - & $0,12 \%$ & $0,21 \%$ & $0,25 \%$ \\
\hline Suzano Hold & - & $0,34 \%$ & $0,80 \%$ & $0,59 \%$ & $0,57 \%$ \\
\hline Suzano Papel & - & - & $0,17 \%$ & $0,21 \%$ & $0,17 \%$ \\
\hline Ultrapar Part $(*)$ & $0,20 \%$ & $0,21 \%$ & $0,20 \%$ & $0,19 \%$ & $0,18 \%$ \\
\hline Legenda $(*) *$ Empro
\end{tabular}

Legenda: (*) - Empresas de Alta Tecnologia

Fonte: Dados da pesquisa.

Entre as companhias que estiveram nas principais colocações do ranking, destacam-se a QGEP, Petro Rio e Ultrapar. Referente as duas primeiras, observa-se uma queda no 
investimento percentual de $\mathrm{P} \& \mathrm{D}$ escalonado pelo ativo, sendo esse um evento que ocorre também com outras companhias. Outro fator em comum, é que ambas as companhias possuem valores de LC e LG elevados nos cinco anos da pesquisa e END baixo até 2015 e maiores em 2016 e 2017. No entanto, a Petro Rio possui uma queda vertiginosa no investimento em P\&D, diferente do que ocorreu no início da análise. Ainda, diferente das empresas citadas, há o caso da Romi, que mesmo possuindo um investimento alto, se comparado as demais empresas brasileiras, não apresenta uma classificação relevante na pesquisa. Contrariamente, a Ultrapar, em nenhum momento contou com um percentual significante de investimento em P\&D, sendo o maior em $2016(0,21 \%)$ e mesmo assim a companhia se fez presente entre as dez melhores empresas do ranking.

Outras companhias com baixo investimento em P\&D foram a Itautec e a Randon Part. A primeira, além de contar com pouco investimento, figurou entre as empresas com indicadores menos representativos do ranking em todos os anos do estudo. A Randon Part, mesmo estando entre as melhores durante um dos anos, não se manteve no patamar. Referente a companhias de maior destaque no cenário brasileiro, como a Embraer e Petrobrás, nota-se que em nenhum momento essas aparecem entre as dez melhores do ranking e, inclusive, a Petrobrás, mesmo possuindo o maior volume de investimento em $\mathrm{P} \& \mathrm{D}$ e, que continua também entre os cinco principais quando escalonado, não possui uma boa classificação (é importante ressaltar o momento da companhia frente a situação em que se encontra).

Portanto, para mensurar se as empresas com os melhores indicadores econômico-financeiros são mais propensas a investir em P\&D, realizou-se o teste de correlação de Spearman.

Tabela 05: Correlação de Spearman.

\begin{tabular}{lccc}
\hline Correlação de Spearman & $\mathbf{N}^{\mathbf{0}}$ de Observações & Rho de Spearman & Significância \\
\hline Ranking TOPSIS & 56 & 0,3047 & $\mathbf{0 , 0 2 2 4}(*)$ \\
\hline Ranking TOPSIS CRITIC & 56 & 0,2969 & $\mathbf{0 , 0 2 6 3 ( * )}$ \\
\hline
\end{tabular}

Legenda: (*) - Significância ao nível de 5\%.

Fonte: Dados da pesquisa.

Conforme o teste de correlação de Spearman, evidencia-se que o Rho é positivo, ou seja, há uma relação crescente entre as variáveis, assim quanto maior o valor atribuído a empresa por meio do ranking gerado a partir do TOPSIS, maior é o investimento percentual em P\&D sobre o ativo total. Esse resultado é encontrado de forma significante em ambos os cálculos do TOPSIS, pelo modelo simples ou por meio dos pesos do CRITIC.

Com base no resultado da correlação de Spearman e da discussão dos indicadores, observa-se que empresas com mais liquidez e com melhor rentabilidade estão associadas ao investimento em P\&D que, por sua vez, a longo prazo, melhora o fluxo de caixa e o resultado da companhia Karjalainen (2008), podendo ser revertido em um investimento maior em P\&D no futuro. De forma a comprovar se de fato, além da relação entre o ranking e o investimento no momento atual, há o efeito da $\mathrm{P} \& \mathrm{D}$ no resultado futuro das companhias, utilizou-se uma regressão linear. O resultado evidencia-se na tabela 06.

Tabela 06: Regressão Linear.

\begin{tabular}{|c|c|}
\hline Variável & $\begin{array}{l}\text { Coeficiente } \\
\text { Sig Var }\end{array}$ \\
\hline Constante & $\begin{array}{l}0,2613 \\
\mathbf{0 , 0 0 2}(*)\end{array}$ \\
\hline P\&D & 3,7780 \\
\hline
\end{tabular}


Investimento em Pesquisa e Desenvolvimento de Empresas de Alta e Média-Alta Tecnologia do Brasil

\begin{tabular}{l|l}
\hline & $\mathbf{0 , 0 5 3}(* *)$ \\
\hline R2 & 0,4017 \\
\hline Significância do modelo & 0,0049 \\
\hline Observações & 49 \\
\hline Leneng &
\end{tabular}

Legenda: $(*)$ - Significância ao nível de 1\%; (**) - Significância ao nível de 10\%. Fonte: Dados da pesquisa.

Percebe-se, por meio da regressão, que a $P \& D$, defasada em dois anos, é significante na explicação do desempenho futuro da companhia, entendida nesse contexto como os indicadores econômico-financeiros da pesquisa. Isso vai ao encontro do resultado obtido por diversos autores como Karjalainen (2008), Eberhart, Maxwell, \& Siddique, (2004) e Huang Hou (2019) que discutem os efeitos positivos do investimento em $\mathrm{P} \& \mathrm{D}$ a longo prazo.

Por conseguinte, percebem-se alguns benefícios relacionados ao investimento em $\mathrm{P} \& \mathrm{D}$, como um fator importante para vantagem competitiva, aumento do poder de mercado (Aghion et al., 2014), relação com o crescimento econômico a longo prazo (Akcigit \& Kerr, 2015) e manutenção de alto padrão de lucro (Love et al., 2009). Além disso, os resultados evidenciam a liquidez e o ROA, indicadores com maior peso no cálculo do ranking, como fatores principais relacionados com o investimento em $\mathrm{P} \& \mathrm{D}$, permitindo generalizar, para o contexto estudado, que empresas que mais investem em $\mathrm{P} \& \mathrm{D}$ optam pelo financiamento interno e possuem retorno maior, diferente das empresas com indicadores menos representativos, que possuem maior endividamento e rentabilidade menor.

\section{CONSIDERAÇÕES FINAIS}

Com o intuito de verificar o investimento $\mathrm{P} \& \mathrm{D}$ das empresas com melhores indicadores econômico-financeiros dos setores de alta e média-alta tecnologia, o estudo desenvolveu um ranking com o método TOPSIS, o qual atribui um valor para cada empresa com base nos indicadores econômico. A amostra totalizou 13 empresas, devido a exclusões realizadas por falta de dados das companhias, principalmente referente a variável de P\&D. Para encontrar a relação entre as empresas com melhores indicadores e investimento em P\&D utilizou-se a correlação de Spearmann.

Os indicadores econômico-financeiros utilizados para a classificação das empresas no ranking foram escolhidos com base na literatura referente a P\&D como fatores importantes. Dessa forma, utilizaram-se no estudo indicadores de Liquidez Corrente e Geral (referente ao financiamento interno), Endividamento (referente ao financiamento externo), ROA e ROE (rentabilidade) e Giro do Ativo (eficiência da gestão dos ativos).

Particularmente, os indicadores de liquidez foram os mais importantes no ranking, principalmente quando da utilização do CRITIC para determinação de pesos conforme a correlação de todos os indicadores. Esse resultado é suportado na literatura, devido ao fluxo de caixa da organização ser um fator fundamental para a continuidade das companhias e, com foco no investimento em $\mathrm{P} \& \mathrm{D}$, está relacionado com o poder de alocação de recursos para essa prática.

Referente a associação com $\mathrm{P} \& \mathrm{D}$, apesar da quantidade de empresas que divulgou essa informação, o resultado atesta que as companhias pertencentes ao grupo mais elevado, ou seja, com o melhores indicadores econômico-financeiros apresentam maior investimento em P\&D escalonado pelo ativo total. Observa-se que essas companhias apresentam liquidez e ROA superiores, os quais dão indícios de benefícios provenientes de investimentos passados em P\&D e que proporcionam a sua continuidade. Além de testar a correlação entre as variáveis, 
verificou-se o efeito da $\mathrm{P} \& \mathrm{D}$ no resultado futuro da companhia, atestando que o investimento em $P \& D$ é positivamente relacionado com os indicadores econômico-financeiros futuros. Dessa forma, por esse investimento associar-se a vantagem competitiva, crescimento econômico a longo prazo e manutenção de alto padrão de lucros, conclui-se que essa prática é boa para a organização, auxiliando-a na continuidade e na melhora dos resultados.

Este estudo contribuiu para a literatura em investimento em $P \& D$, aumentando a sua relação com os indicadores econômico-financeiros e, na prática, mostrando que esse investimento está relacionado com as empresas que possuem a melhor classificação em um ranking baseado nesses indicadores. Além, é claro, de averiguar um cenário de companhias diretamente relacionadas a $P \& D$ e indicar a sua tendência quanto a esse investimento. Assim, por meio dos resultados, torna-se interessante para os stakeholders visualizar se a companhia investe nessa prática como forma de melhorar os resultados futuros. Para pesquisas futuras, torna-se interessante comparar outros cenários, de forma a entender a diferença entre os próprios setores ou entre países e, de forma a melhorar a generalização dos resultados, elaborando o estudo em um cenário que a P\&D seja mais divulgada.

Apesar das importantes contribuições deste estudo, há certas limitações em que identificam-se os seguintes pontos: a consideração de quais gastos são relacionados a P\&D é difícil, caracterizando-se, normalmente, como uma escolha discricionária do gerente (Koh \& Reeb, 2015); empresas com divulgação dos valores referente a P\&D em branco, dessa maneira, não há essa informação nos relatórios contábeis e, assim, não é possível saber se foi uma decisão consciente de não registrar esse tipo de investimento ou não (Mcvay, 2006), portanto, as diferenças entre o modo que as companhias registram e divulgam a $\mathrm{P} \& \mathrm{D}$ podem ter efeito nos resultados da pesquisa; e pela amostra de empresas com divulgação de $\mathrm{P} \& \mathrm{D}$ afetar de forma assimétrica quantidade da população na análise da diferença no investimento em $\mathrm{P} \& \mathrm{D}$, pode ser que haja um viés no resultado.

\section{REFERÊNCIAS}

Aghion, P., Bechtold, S., Cassar, L., \& Herz, H. (2014). The causal effects of competition on innovation: experimental evidence. National Bureau of Economic Research.

Akcali, B. Y., \& Sismanoglu, E. (2015). Innovation and the Effect of Research and Development (R\&D) Expenditure on Growth in Some Developing and Developed Countries. Procedia - Social and Behavioral Sciences, 195, 768-775. https://doi.org/10.1016/j.sbspro.2015.06.474

Akcigit, U., \& Kerr, W. (2015). Growth through Heterogeneous Innovations. Journal of Political Economy, 126(4), 1374-1443.

Assaf Neto, A. (2014). Finanças corporativas e valor (7th ed.). São Paulo: Atlas S.A.

Balcerzak, A. P., \& Pietrzak, M. B. (2016). Application of TOPSIS Method for Analysis of Sustainable Development in European Union Countries. In The 10th International Days of Statistics and Economics. Conference Proceedings. (pp. 82-92).

Beuren, I. M. (2004). Como elaborar trabalhos monográficos em contabilidade: teoria e prática. São Paulo: Atlas.

Brown, J. R., Martinsson, G., \& Petersen, B. C. (2012). Do financing constraints matter for R\&D? European Economic Review, 56(8), 1512-1529. https://doi.org/10.1016/j.euroecorev.2012.07.007

Brown, J. R., \& Petersen, B. C. (2011). Cash holdings and R\&D smoothing. Journal of Corporate Finance, 17(3), 694-709. https://doi.org/10.1016/j.jcorpfin.2010.01.003

Brown, J. R., \& Petersen, B. C. (2015). Which investments do firms protect? Liquidity management and real adjustments when access to finance falls sharply. Journal of 
Financial Intermediation, 24(4), 441-465. https://doi.org/10.1016/j.jfi.2014.03.002

Coad, A. (2018). Persistent heterogeneity of R\&D intensities within sectors: Evidence and policy implications. Research Policy. https://doi.org/10.1016/j.respol.2018.07.018

Collis, J., \& Hussey, R. (2005). Pesquisa em Administração: Um guia prático para alunos de graduação e pós-graduação (2. ed.). Porto Alegre: Bookman.

Cornell, B., \& Shapiro, A. C. (1988). Financing Corporate Growth. Journal of Applied Corporate Finance, 1(2), 6-22. https://doi.org/10.1108/02756660610649994

Cowling, M., Liu, W., Ledger, A., \& Zhang, N. (2015). What really happens to small and medium-sized enterprises in a global economic recession? UK evidence on sales and job dynamics. International Small Business Journal, 33(5), 488-513. https://doi.org/10.1177/0266242613512513

Degenhart, L., Vogt, M., Da Rosa, F. S., \& Fank, O. S. (2015). Ranking de evidências dos impactos ambientais de empresas brasileiras: um comparativo entre métodos multicritérios T-ODA e TOPSIS. In XVII ENGEMA - Encontro Internacional sobre Gestão Empresarial e meio Ambiente. São Paulo.

Diakoulaki, D., Mavrotas, G., \& Papayannakis, L. (1995). Determining objective weights in multiple criteria problems: The critic method. Computers and Operations Research, 22(7), 763-770. https://doi.org/10.1016/0305-0548(94)00059-H

Doh, S., \& Kim, B. (2014). Government support for SME innovations in the regional industries: The case of government financial support program in South Korea. Research Policy, 43(9), 1557-1569. https://doi.org/10.1016/j.respol.2014.05.001

Eberhart, A. C., Maxwell, W. F., \& Siddique, A. R. (2004). An Examination of Long-Term Abnormal Stock Returns and Operating Performance Following R\&d Increases. Journal of Finance, 59(2), 623-650. https://doi.org/10.1111/j.1540-6261.2004.00644.x

Gonçalves, W. D. B., \& Lemes, S. (2018). A relação dos gastos com P\&D com a qualidade da informação contábil. Revista Contabilidade Vista \& Revista, 29(2), 68-95. https://doi.org/10.1016/j.agee.2006.02.009

Guney, Y., Karpuz, A., \& Ozkan, N. (2017). R\&D investments and credit lines. Journal of Corporate Finance, 46, 261-283. https://doi.org/10.1016/j.jcorpfin.2017.07.011

Guo, D., Guo, Y., \& Jiang, K. (2017). Funding Forms, Market Conditions, and Dynamic Effects of Government R\&D Subsidies: Evidence From China. Economic Inquiry, 55(2), 825842. https://doi.org/10.1111/ecin.12395

Hauke, J., \& Kossowski, T. (2011). Comparison of Values of Pearson's and Spearman's Correlation Coefficients on the Same Sets of Data. Quaestiones Geographicae, 30(2), 8793. https://doi.org/10.2478/v10117-011-0021-1

Hein, N., Degenhart, L., Vogt, M., Kroenke, A., \& Campestrini, I. M. (2015). Método TOPSIS na avaliação das empresas listadas no IBRX-100: uma avaliação multicritério dos impactos ambientais. In CONBREPRO - Congresso Brasileiro de Engenharia de Produção. Ponta Grossa - PR.

Holmström, B., \& Tirole, J. (1998). Private and Public Supply of Liquidity. Journal of Political Economy, 106(1), 1-40. https://doi.org/10.1080/15732479.2013.833949

Huang, C., \& Hou, T. C. (2019). Innovation, research and development, and firm profitability in Taiwan: Causality and determinants. International Review of Economics \& Finance, 59, 385-394. https://doi.org/10.1016/j.iref.2018.10.004

IBGE. (2003). Análise dos resultados. Retrieved November 15, 2018, from https://ww2.ibge.gov.br/home/estatistica/economia/industria/pia/empresas/comentario20 03.pdf

Iudícibus, I. (2012). Análise de balanços (6th ed.). São Paulo: Atlas S.A.

Jung, H., Hwang, J., \& Kim, B. K. (2018). Does R\&D investment increase SME survival during a recession? Technological Forecasting and Social Change, 137, 190-198. 
https://doi.org/10.1016/j.techfore.2018.07.042

Kang, T., Baek, C., \& Lee, J. (2017). The persistency and volatility of the fi rm R \& D investment: Revisited from the perspective of technological capability. Research Policy Journal, 46, 1570-1579. https://doi.org/10.1016/j.respol.2017.07.006

Karjalainen, P. (2008). R\&D investments: The effects of different financial environments on firm profitability. Journal of Multinational Financial Management, 18(2), 79-93. https://doi.org/10.1016/j.mulfin.2007.02.006

Koh, P. S., \& Reeb, D. (2015). Missing R\&D. Journal of Accounting and Economics, 60, 73 94. https://doi.org/10.1016/j.jacceco.2015.03.004

Landry, S., \& Callimaci, A. (2003). The effect of management incentives and cross-listing status on the accounting treatment of R\&D spending. Journal of International Accounting, Auditing and Taxation, 12(2), 131-152. https://doi.org/10.1016/j.intaccaudtax.2003.08.003

Love, J. H., Roper, S., \& Du, J. (2009). Innovation, ownership and profitability. International Journal of Industrial Organization, 27(3), 424-434. https://doi.org/10.1016/j.ijindorg.2008.11.001

Marion, J. C. (2012). Analise das demonstrações contábeis. São Paulo: Atlas S.A.

MCTI. (2016). Ministério da Ciência, Tecnologia e Inovação. Retrieved December 6, 2018, from http://www.mcti.gov.br

Mcvay, S. E. (2006). Earnings Management Using Classification Shifting: An Examination of Core Earnings and Special Items. The Accounting Review, 81(3), 501-531. https://doi.org/10.2308/accr.2006.81.3.501

Mukaka, M. M. (2012). Statistics Corner: A guide to appropriate use of Correlation coefficient in medical research. Malawi Medical Journal, 24(3), 69-71. https://doi.org/10.1016/j.cmpb.2016.01.020

Opler, T. C., \& Titman, S. (1994). Financial Distress and Corporate Performance. The Journal of Finance, 49(3), 1015-1040. https://doi.org/10.1111/j.1540-6261.1994.tb00086.x

Radas, S., Anić, I. D., Tafro, A., \& Wagner, V. (2015). The effects of public support schemes on small and medium enterprises. Technovation, 38, 15-30. https://doi.org/10.1016/j.technovation.2014.08.002

Tonolli, B. B., Rover, S., \& Ferreira, D. D. M. (2017). Influência dos investimentos ambientais e dos indicadores econômico-financeiros na seleção de empresas para compor o Índice De Sustentabilidade Empresarial (ISE). Revista Catarinense Da Ciência Contábil, 16(48), 69-85. https://doi.org/10.16930/2237-7662/rccc.v16n48.2315

Ugur, M., Trushin, E., \& Solomon, E. (2016). Inverted-U relationship between R\&D intensity and survival: Evidence on scale and complementarity effects in UK data. Research Policy, 45(7), 1474-1492. https://doi.org/10.1016/j.respol.2016.04.007

Viglioni, M. T. D., Carvalho, F. de M., Benedicto, G. C. de, \& Do Prado, J. W. (2018). Indicadores Econômico-Financeiros Determinantes de Fusões e Aquisições: um Estudo na Indústria de Tecnologia no Brasil. Contabilidade, Gestão e Governança, 21(3), 420-438. https://doi.org/10.21714/1984-3925_2018v21n3a7

Yoon, K. P., \& Hwang, C. L. (1995). Multiple attribute decision making: an introduction. Sage Publication. 